Supporting Information for

\title{
Probing the Dipole-Bound State in the 9-Phenanthrolate Anion by Photodetachment Spectroscopy, Resonant Two-Photon Photoelectron Imaging, and Resonant Photoelectron Spectroscopy
}

Dao-Fu Yuan, Yue-Rou Zhang, Chen-Hui Qian, Yuan Liu ${ }^{\dagger}$, and Lai-Sheng Wang*

Department of Chemistry, Brown University, Providence, Rhode Island 02912, United States

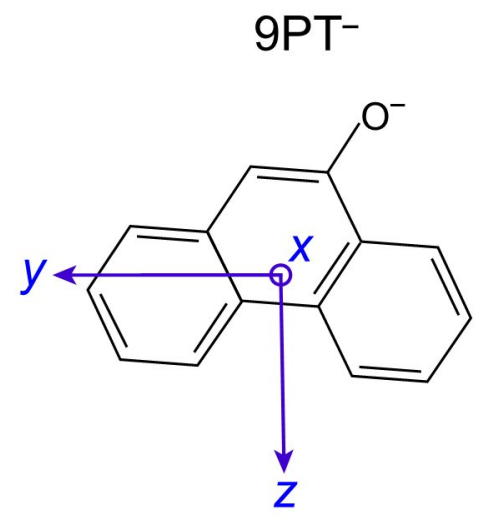

$C_{\mathrm{s}}$

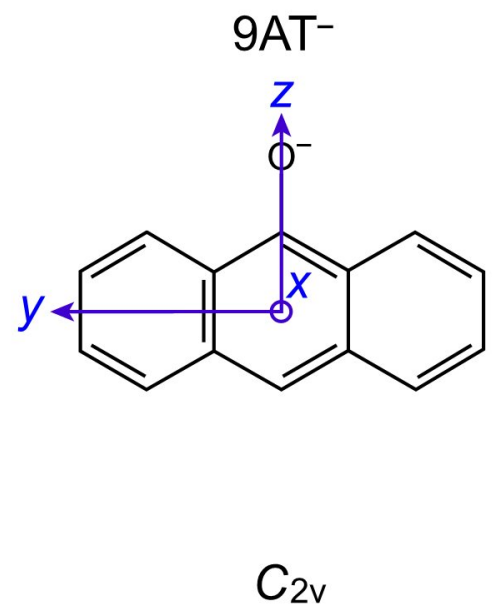

$\mathrm{C}_{2 \mathrm{v}}$

Figure S1. The structures, the coordinates used., and symmetries of both $9 \mathrm{PT}^{-}$and $9 \mathrm{AT}^{-}$. 

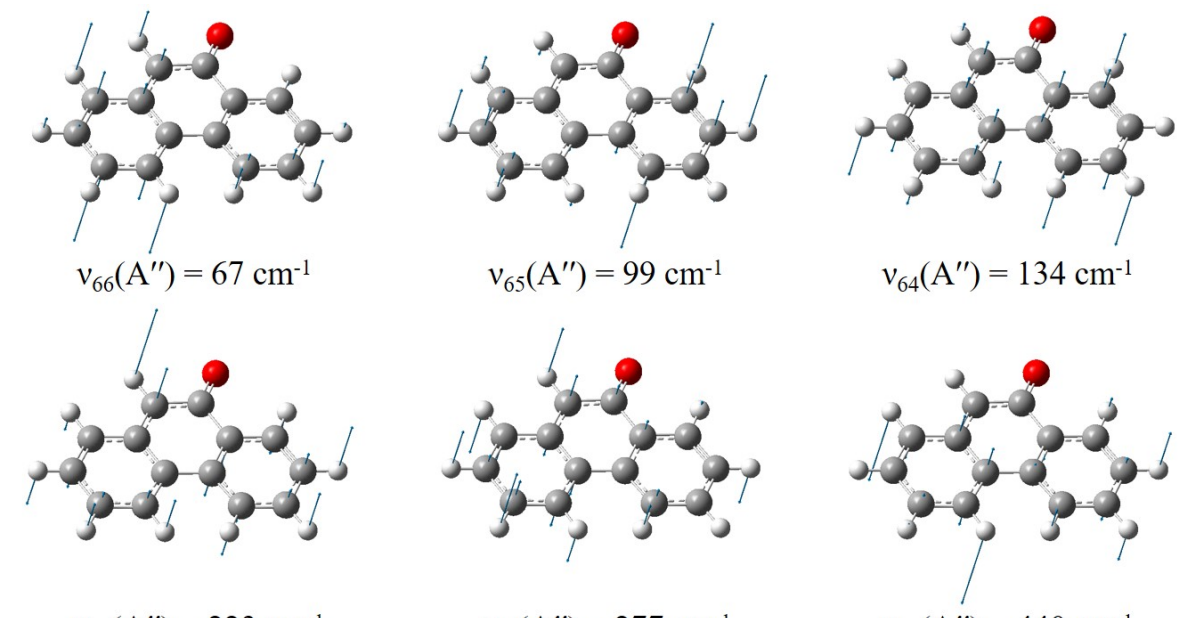

$$
v_{63}\left(A^{\prime \prime}\right)=223 \mathrm{~cm}^{-1}
$$

$$
v_{62}\left(\mathrm{~A}^{\prime \prime}\right)=277 \mathrm{~cm}^{-1}
$$

$v_{60}\left(A^{\prime \prime}\right)=440 \mathrm{~cm}^{-1}$
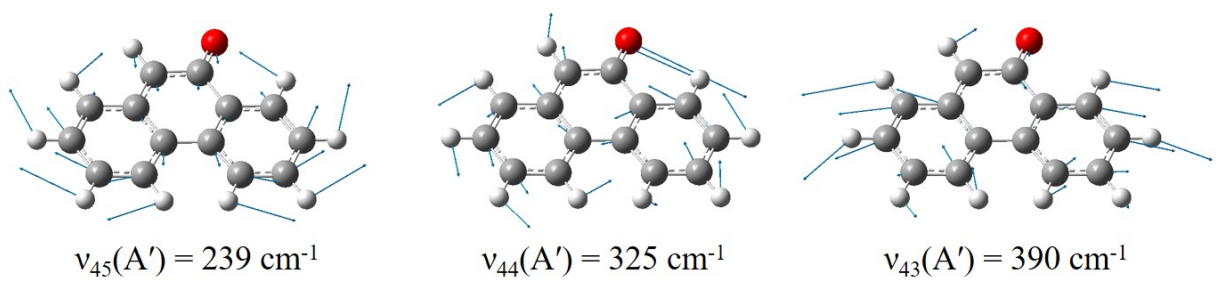

Figure S2. The atomic displacement vectors of the vibrational modes observed for 9PT. The symmetries and the measured frequencies are shown below for each mode. 
Table S1. Exponents of the diffuse basis functions used in the calculation on the three carbon atoms circled in the molecular structure.

\begin{tabular}{|c|c|}
\hline \multirow{2}{*}{$4 \mathrm{~s}$} & 0.03942165641 \\
\cline { 2 - 2 } & 0.01633033298 \\
\cline { 2 - 2 } & 0.00676480391 \\
\cline { 2 - 2 } & $2.80230488 \mathrm{E}-3$ \\
\hline \multirow{2}{*}{$3 \mathrm{p}$} & 0.03501152294 \\
\cline { 2 - 2 } & 0.01218880611 \\
\hline \multirow{2}{*}{$2 \mathrm{~d}$} & $4.2433742354 \mathrm{E}-3$ \\
\cline { 2 - 2 } & 0.0921823154 \\
\hline \multirow{2}{*}{$1 \mathrm{f}$} & $2.6721947401 \mathrm{E}-2$ \\
\hline
\end{tabular}

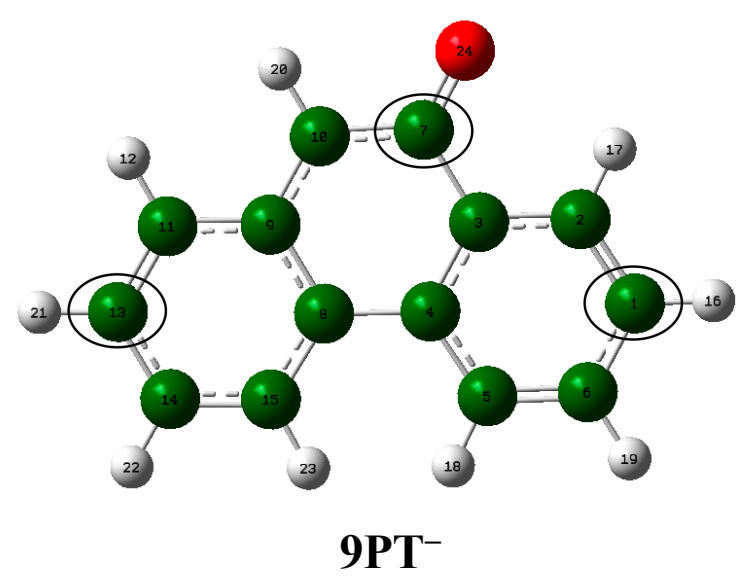


Table S2. The theoretical frequencies of 9PT at the DFT/ B3LYP/6-311++ (d, p) level of theory.

\begin{tabular}{|c|c|c|c|c|c|}
\hline Mode & Frequency $\left(\mathrm{cm}^{-1}\right)$ & Symmetry & Mode & Frequency $\left(\mathrm{cm}^{-1}\right)$ & Symmetry \\
\hline$v_{1}$ & 3209 & \multirow{33}{*}{$\mathrm{A}^{\prime}$} & $v_{34}$ & 1013 & \\
\hline$v_{2}$ & 3201 & & $v_{35}$ & 894 & \\
\hline$v_{3}$ & 3196 & & $v_{36}$ & 794 & \\
\hline$v_{4}$ & 3192 & & $v_{37}$ & 719 & \\
\hline$v_{5}$ & 3185 & & $v_{38}$ & 665 & \\
\hline$v_{6}$ & 3184 & & $v_{39}$ & 629 & \\
\hline$v_{7}$ & 3177 & & $v_{40}$ & 611 & \\
\hline$v_{8}$ & 3170 & & $v_{41}$ & 502 & \\
\hline$v_{9}$ & 3169 & & $v_{42}$ & 425 & \\
\hline$v_{10}$ & 1642 & & $v_{43}$ & 398 & \\
\hline$v_{11}$ & 1619 & & $v_{44}$ & 328 & \\
\hline$v_{12}$ & 1604 & & $v_{45}$ & 242 & \\
\hline$v_{13}$ & 1569 & & $v_{46}$ & 1009 & \multirow{21}{*}{$\mathrm{A}^{\prime \prime}$} \\
\hline$v_{14}$ & 1550 & & $v_{47}$ & 989 & \\
\hline$v_{15}$ & 1524 & & $v_{48}$ & 978 & \\
\hline$v_{16}$ & 1500 & & $v_{49}$ & 960 & \\
\hline$v_{17}$ & 1478 & & $v_{50}$ & 885 & \\
\hline$v_{18}$ & 1454 & & $v_{51}$ & 871 & \\
\hline$v_{19}$ & 1418 & & $v_{52}$ & 844 & \\
\hline$v_{20}$ & 1358 & & $v_{53}$ & 781 & \\
\hline$v_{21}$ & 1344 & & $v_{54}$ & 778 & \\
\hline$v_{22}$ & 1342 & & $v_{55}$ & 747 & \\
\hline$v_{23}$ & 1297 & & $v_{56}$ & 729 & \\
\hline$v_{24}$ & 1296 & & $v_{57}$ & 654 & \\
\hline$v_{25}$ & 1254 & & $v_{58}$ & 557 & \\
\hline$v_{26}$ & 1208 & & $v_{59}$ & 494 & \\
\hline$v_{27}$ & 1186 & & $v_{60}$ & 443 & \\
\hline$v_{28}$ & 1181 & & $v_{61}$ & 421 & \\
\hline$v_{29}$ & 1160 & & $v_{62}$ & 283 & \\
\hline$v_{30}$ & 1131 & & $v_{63}$ & 226 & \\
\hline$v_{31}$ & 1103 & & $v_{64}$ & 140 & \\
\hline$v_{32}$ & 1062 & & $v_{65}$ & 93 & \\
\hline$v_{33}$ & 1058 & & $v_{66}$ & 68 & \\
\hline
\end{tabular}


TABLE S3. The excitation energy of the triplet valence excited state computed for 9PT- ${ }^{-}$at the B3LYP level, as well as the main electronic configurations. The HOMO and LUMO involved in the VE are shown below the Table (isovalue $=0.02$ ).

\begin{tabular}{ccc}
\hline \hline & Excitation Energy $(\mathrm{eV})$ & Electronic Configuration \\
\hline Triplet excited state & 2.1 & $\ldots 43 \mathrm{a}^{\prime 2} 8 \mathrm{a}^{\prime \prime} 9 \mathrm{a}^{\prime \prime}$ \\
\hline Ground 9PT & 0 & $\ldots 43 \mathrm{a}^{\prime 2} 8 \mathrm{a}^{\prime \prime 2} 9 \mathrm{a}^{\prime \prime}$ \\
\hline \hline
\end{tabular}

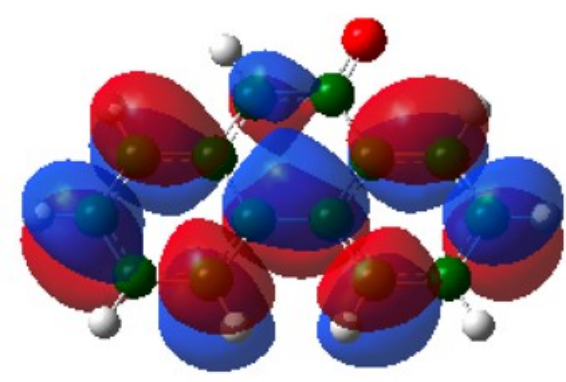

$9 \mathrm{a}^{\prime \prime}$, LUMO of the anion involved in the valence bound excited state

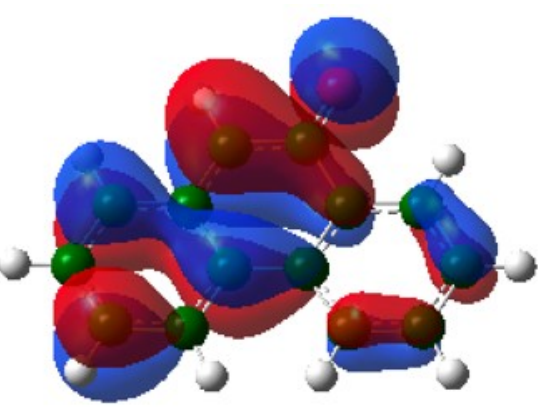

$8 \mathrm{a}^{\prime \prime}, \mathrm{HOMO}$ of the anion 